\title{
Reduction of Low-Thrust Continuous Controls for Trajectory Dynamics
}

\author{
Jennifer S. Hudson* \\ University of Michigan, Ann Arbor, Michigan 48019 \\ and \\ Daniel J. Scheeres \pm \\ University of Colorado, Boulder, Colorado 80309 \\ DOI: $\underline{10.2514 / 1.40619}$
}

\begin{abstract}
A novel method to evaluate the trajectory dynamics of low-thrust spacecraft is developed. The thrust vector components are represented as Fourier series in eccentric anomaly, and Gauss's variational equations are averaged over one orbit to define a set of secular equations. These secular equations are a function of only 14 of the thrust Fourier coefficients, regardless of the order of the original Fourier series, and are sufficient to accurately determine a low-thrust spiral trajectory with significantly reduced computational requirements as compared with integration of the full Newtonian problem. This method has applications to low-thrust spacecraft targeting and optimal control problems.
\end{abstract}

\section{Introduction}

$\mathbf{L}$ OW-THRUST propulsion systems offer an efficient option for many interplanetary and Earth-orbit missions. However, optimal control of these systems can pose a difficult design challenge. Analytical or approximate solutions exist for several special cases of optimal low-thrust orbit-transfer problems, but the general continuous-thrust problem requires full numerical integration of each initial condition and thrust profile. The trajectory is highly sensitive to these variables; thus, the optimal control law over tens or hundreds of orbits of a spiral trajectory is often difficult to determine.

Analytical solutions have been developed for many special-case transfers, such as the logarithmic spiral [1-3], Forbes's spiral [1], the exponential sinusoid $[4,5]$, the case of constant radial or circumferential thrust [6- $\underline{-} \overline{8}]$, Markopoulos's Keplerian thrust programs [9], Lawden's spiral [10], and Bishop and Azimov's spiral [11]. More recent solutions have used the calculus of variations [12] or direct optimization methods [13] to determine optimal low-thrust control laws within certain constraints. Several methods for open-loop minimum-time transfers [14-16] and optimal transfers using Lyapunov feedback control [17,18] also exist. Averaging methods in combination with other approaches have proven to be effective at overcoming sensitivities to small variations in the initial orbit and thrust profile [19-22], yet all solutions remain limited to certain regions of the thrust and orbital parameter space.

The focus of this study is a novel method to evaluate the effect of low-thrust propulsion on spacecraft orbit dynamics with minimal constraints. We represent each component of the thrust acceleration as a Fourier series in eccentric anomaly and then average Gauss's variational equations over one orbit to define a set of secular equations. The equations are a function of only 14 of the thrust Fourier coefficients, regardless of the order of the original Fourier series; thus, the full continuous control is reduced to a set of 14 parameters.

Presented as Paper 6617 at the AIAA/AAS Astrodynamics Specialist Conference and Exhibit, Honolulu, HI, 18-21 August 2008; received 26 August 2008; accepted for publication 27 January 2009. Copyright $@ 2009$ by Jennifer Hudson. Published by the American Institute of Aeronautics and Astronautics, Inc., with permission. Copies of this paper may be made for personal or internal use, on condition that the copier pay the $\$ 10.00$ per-copy fee to the Copyright Clearance Center, Inc., 222 Rosewood Drive, Danvers, MA 01923; include the code 0731-5090/09 \$10.00 in correspondence with the CCC.

*Ph.D. Candidate, Department of Aerospace Engineering.

${ }^{\dagger}$ A. Richard Seebass Professor, Aerospace Engineering Sciences, Colorado Center for Astrodynamics Research. Associate Fellow AIAA.
With the addition of a small correction term to eliminate offsets of the averaged trajectory due to initial conditions, the averaged secular equations are sufficient to determine a low-thrust trajectory with a high degree of accuracy. This is verified by comparison of the averaged trajectory dynamics with the fully integrated Newtonian equations of motion for a basic step-acceleration function and a randomly generated continuous-acceleration function.

The method has certain limits of applicability. First, the thrust acceleration must be able to be represented by a Fourier series, as is true for almost any physical system. The acceleration must be periodic (not necessarily with a period of one orbit) with sufficiently low magnitude that the orbit shape does not change significantly from start to finish. The orbit may closely approach zero eccentricity, but the current method cannot tolerate exactly circular orbits.

The focus of the current paper is to introduce this methodology and present numerical justification of the result in some limited settings. Subsequent work will demonstrate the use of these secular equations in solving orbit-transfer problems for low-thrust spacecraft and will study the results for near-circular orbits.

\section{Variational Equations}

We consider a spacecraft of negligible mass in orbit about a central body, which is assumed to be a point mass. The spacecraft is subject to a continuous thrust acceleration of potentially varying magnitude and direction. The spacecraft trajectory can be described by the Newtonian equations of motion:

$$
\begin{gathered}
\dot{\mathbf{r}}=\mathbf{v} \\
\dot{\mathbf{v}}=-\frac{\mu}{r^{3}} \mathbf{r}+\mathbf{F}
\end{gathered}
$$

where $\mathbf{r}$ is the position vector, $\mathbf{v}$ is the velocity vector, and $\mu$ is the standard gravitational parameter of the central body. The thrustacceleration vector $\mathbf{F}$ can be resolved along the radial, normal, and circumferential directions:

$$
\mathbf{F}=F_{R} \hat{\mathbf{r}}+F_{W} \hat{\mathbf{w}}+F_{S}(\hat{\mathbf{w}} \times \hat{\mathbf{r}})
$$

where $\hat{\mathbf{r}}=\mathbf{r} /|\mathbf{r}|$ and $\hat{\mathbf{w}}=(\mathbf{r} \times \mathbf{v}) /(|\mathbf{r} \times \mathbf{v}|)$. The Newtonian equations can be decomposed into the Lagrange planetary equations, which describe the time rate of change of the classical orbit elements of a body subject to the $F_{R}, F_{W}$, and $F_{S}$ perturbations. The Gaussian form of the Lagrange planetary equations is presented next [23] : 


$$
\begin{gathered}
\frac{d a}{d t}=2 \sqrt{\frac{a}{\mu}}\left[F_{R} \frac{a e}{\sqrt{1-e^{2}}} \sin v+F_{S} \frac{a^{2} \sqrt{1-e^{2}}}{a(1-e \cos E)}\right] \\
\frac{d e}{d t}=\sqrt{\frac{a}{\mu}} \sqrt{1-e^{2}}\left[F_{R} \sin v+F_{S}(\cos v+\cos E)\right] \\
\frac{d i}{d t}=\sqrt{\frac{a}{\mu}} \frac{(1-e \cos E)}{\sqrt{1-e^{2}}} F_{W} \cos (v+\omega) \\
\frac{d \Omega}{d t}=\sqrt{\frac{a}{\mu} \frac{(1-e \cos E)}{\sqrt{1-e^{2}}} F_{W} \sin (v+\omega)} \\
+\sqrt{\frac{a}{\mu}} \frac{\sqrt{1-e^{2}}}{e}\left[-F_{R} \cos v+F_{S}\left(1+\frac{1-e \cos E}{1-e^{2}}\right) \sin v\right] \\
-\cos i \frac{d \Omega}{d t} \\
+2 \sqrt{1-e^{2}} \sin ^{2}\left(\frac{i}{2}\right) \dot{\Omega} \\
=-2 \sqrt{\frac{a}{\mu}}(1-e \cos E) F_{R}+\left(1-\sqrt{1-e^{2}}\right)(\dot{\omega}+\dot{\Omega})
\end{gathered}
$$

where $a$ is the semimajor axis, $e$ is the eccentricity, $i$ is the inclination, $\Omega$ is the longitude of the ascending node, $\omega$ is the argument of periapsis, $v$ is the true anomaly, $E$ is the eccentric anomaly, and $\epsilon_{1}+\int n \mathrm{~d} t=l$ is the mean longitude. The mean anomaly is the difference of the mean longitude and the longitude of periapsis:

$$
M=\int n \mathrm{~d} t+\epsilon_{1}-(\Omega+\omega)
$$

In the modeling and simulation of low-thrust spacecraft orbits, both the Newtonian equations and the Gauss equations provide identical results. The Gauss equations are often preferred for clear visualization of the orbit over time.

\section{Fourier Series Expansion of Control Law}

According to Fourier's theorem, any piecewise-smooth function $f(\theta)$ with a finite number of jump discontinuities on the interval $(0, L)$ can be represented by a Fourier series that converges to the periodic extension of the function itself [24]:

$$
f(\theta) \sim \sum_{k=0}^{\infty}\left[a_{k} \cos \left(\frac{2 \pi k \theta}{L}\right)+b_{k} \sin \left(\frac{2 \pi k \theta}{L}\right)\right]
$$

When jump discontinuities exist, the Fourier series converges to the average of the two limits. For an interval of interest $L=m \pi$, the Fourier coefficients are found by

$$
\begin{gathered}
a_{0}=\frac{1}{m \pi} \int_{0}^{m \pi} f(\theta) d \theta \\
a_{k}=\frac{2}{m \pi} \int_{0}^{m \pi} f(\theta) \cos \left(\frac{2 k \theta}{m}\right) d \theta \\
b_{k}=\frac{2}{m \pi} \int_{0}^{m \pi} f(\theta) \sin \left(\frac{2 k \theta}{m}\right) d \theta
\end{gathered}
$$

Nearly all physical systems meet the conditions of piecewise smoothness and jump discontinuities; thus, this representation can be applied to almost any general low-thrust spacecraft control law. Given an arbitrary acceleration vector $\mathbf{F}$, each component can be represented as a Fourier series over an arbitrary time interval. The Fourier series can be expanded in time or in a time-varying orbital parameter, such as true anomaly, eccentric anomaly, or mean anomaly. Let $\theta$ represent this arbitrary parameter:

$$
\begin{gathered}
F_{R}=\sum_{k=0}^{\infty}\left[\alpha_{k}^{R, \theta} \cos \left(\frac{2 \pi k \theta}{L}\right)+\beta_{k}^{R, \theta} \sin \left(\frac{2 \pi k \theta}{L}\right)\right] \\
F_{W}=\sum_{k=0}^{\infty}\left[\alpha_{k}^{W, \theta} \cos \left(\frac{2 \pi k \theta}{L}\right)+\beta_{k}^{W, \theta} \sin \left(\frac{2 \pi k \theta}{L}\right)\right] \\
F_{S}=\sum_{k=0}^{\infty}\left[\alpha_{k}^{S, \theta} \cos \left(\frac{2 \pi k \theta}{L}\right)+\beta_{k}^{S, \theta} \sin \left(\frac{2 \pi k \theta}{L}\right)\right]
\end{gathered}
$$

The acceleration function is thus defined by the coefficients $\alpha_{k}^{D, \theta}$ and $\beta_{k}^{D, \theta}$, where the superscripts $D$ and $\theta$ indicate the acceleration direction and the expansion variable, respectively.

\section{Averaged Variational Equations}

We begin our first-order averaging analysis by assuming an acceleration vector that is to be specified over one orbit period $(L=2 \pi)$ with a sufficiently low magnitude that the size and shape of the orbit does not change significantly over one revolution. Therefore, we may average the Gauss equations over one orbit period with respect to mean anomaly to find equations for the mean orbit elements:

$$
\overline{\dot{\mathfrak{e}}}=\frac{1}{2 \pi} \int_{0}^{2 \pi} \dot{\propto} d M
$$

where $\propto$ represents any orbit element. Our $2 \pi$ periodic-orbit assumption slightly simplifies the Fourier series and coefficient definitions:

$$
\begin{gathered}
F_{R}=\sum_{k=0}^{\infty}\left[\alpha_{k}^{R, \theta} \cos k \theta+\beta_{k}^{R, \theta} \sin k \theta\right] \\
F_{W}=\sum_{k=0}^{\infty}\left[\alpha_{k}^{W, \theta} \cos k \theta+\beta_{k}^{W, \theta} \sin k \theta\right] \\
F_{S}=\sum_{k=0}^{\infty}\left[\alpha_{k}^{S, \theta} \cos k \theta+\beta_{k}^{S, \theta} \sin k \theta\right]
\end{gathered}
$$

$$
\alpha_{0}^{D, \theta}=\frac{1}{2 \pi} \int_{0}^{2 \pi} F(\theta) d \theta
$$

$$
\begin{aligned}
& \alpha_{k}^{D, \theta}=\frac{1}{\pi} \int_{0}^{2 \pi} F(\theta) \cos (k \theta) d \theta \\
& \beta_{k}^{D, \theta}=\frac{1}{\pi} \int_{0}^{2 \pi} F(\theta) \sin (k \theta) d \theta
\end{aligned}
$$

At this point, the choice of orbital parameter for the thrustacceleration vector components' Fourier series expansion becomes significant. If the acceleration components are expanded as Fourier series in true anomaly and the independent parameter for the 
averaging is likewise shifted to true anomaly, the resulting secular equations become quite lengthy and complex. For example, the equation for the semimajor axis becomes

$$
\begin{aligned}
\overline{\dot{a}}= & \frac{1}{2 \pi} \int_{0}^{2 \pi} \dot{a} d M=\frac{1}{2 \pi}\left(1-e^{2}\right)^{\frac{3}{2}} \int_{0}^{2 \pi} \frac{\dot{a}}{(1+e \cos v)^{2}} d v \\
= & \frac{1}{\pi} \sqrt{\frac{a}{\mu}}\left(1-e^{2}\right) \int_{0}^{2 \pi}\left[F_{R} \frac{a e \sin v}{(1+e \cos v)^{2}}+F_{S} \frac{a}{1+e \cos v}\right] d v \\
= & \frac{1}{\pi} \sqrt{\frac{a}{\mu}}\left(1-e^{2}\right) \int_{0}^{2 \pi} \\
& \times\left[\left(\sum_{k=0}^{\infty} \alpha_{k}^{R, v} \cos k v+\beta_{k}^{R, v} \sin k v\right) \frac{a e \sin v}{(1+e \cos v)^{2}}\right. \\
& \left.+\left(\sum_{k=0}^{\infty} \alpha_{k}^{S, v} \cos k v+\beta_{k}^{S, v} \sin k v\right) \frac{a}{1+e \cos v}\right] d v
\end{aligned}
$$

Note that the denominator $(1+e \cos v)^{k}$ can be expanded as a cosine series in its own right:

$$
\frac{1}{(1+e \cos v)^{k}}=\sum_{i=0}^{\infty} b_{i}^{k}(e) \cos i v
$$

Thus, in the true anomaly expansion, each averaged equation contains integrals of products of the sine and cosine series. Resolved into secular equations, each equation will contain the full Fourier series for each relevant acceleration direction. Even more complicated results are found if the expansion and averaging are carried out in mean anomaly.

However, if the acceleration vector components are expanded as Fourier series in eccentric anomaly and the averaging is carried out with eccentric anomaly as the independent parameter, the problematic denominators are eliminated, as $d M=$ $(1-e \cos E) d E$ :

$$
\overline{\dot{\propto}}=\frac{1}{2 \pi} \int_{0}^{2 \pi}(1-e \cos E) \dot{œ} d E
$$

Applying this, the averaged Gauss equations with respect to eccentric anomaly can be stated as

$$
\begin{aligned}
\overline{\dot{a}}=\frac{1}{\pi} \sqrt{\frac{a^{3}}{\mu}} \int_{0}^{2 \pi}\left[F_{R} e \sin E+F_{S} \sqrt{1-e^{2}}\right] d E \\
\overline{\dot{e}}=\frac{1}{2 \pi} \sqrt{\frac{a}{\mu}} \sqrt{1-e^{2}} \int_{0}^{2 \pi} \\
\times\left[F_{R} \sqrt{1-e^{2}} \sin E+F_{S}\left(2 \cos E-\frac{3}{2} e-\frac{1}{2} e \cos 2 E\right)\right] d E \\
\bar{i}=\frac{1}{2 \pi} \sqrt{\frac{a}{\mu}} \frac{1}{\sqrt{1-e^{2}}} \int_{0}^{2 \pi} F_{W} \\
\times\left[\left(1+e^{2}\right) \cos \omega \cos E-\frac{3}{2} e \cos \omega-\sqrt{1-e^{2}} \sin \omega \sin E\right. \\
\left.-\frac{1}{2} e \cos \omega \cos 2 E+\frac{1}{2} e \sqrt{1-e^{2}} \sin \omega \sin 2 E\right] d E
\end{aligned}
$$

$$
\begin{aligned}
\overline{\dot{\Omega}} & =\frac{1}{2 \pi} \sqrt{\frac{a}{\mu}} \frac{\csc i}{\sqrt{1-e^{2}}} \int_{0}^{2 \pi} F_{W} \\
& \times\left[\sqrt{1-e^{2}} \cos \omega \sin E+\left(1+e^{2}\right) \sin \omega \cos E-\frac{3}{2} e \sin \omega\right. \\
& \left.-\frac{1}{2} e \sqrt{1-e^{2}} \cos \omega \sin 2 E-\frac{1}{2} e \sin \omega \cos 2 E\right] d E \\
\overline{\dot{\omega}} & =\frac{1}{2 \pi} \sqrt{\frac{a}{\mu}} \frac{1}{e} \int_{0}^{2 \pi}\left[-F_{R}\left(\sqrt{1-e^{2}} \cos E-e \sqrt{1-e^{2}}\right)\right. \\
& \left.+F_{S}\left(\left(2-e^{2}\right) \sin E-\frac{1}{2} e \sin 2 E\right)\right] d E-\cos i \overline{\dot{\Omega}}
\end{aligned}
$$

$$
\begin{aligned}
\overline{\dot{\epsilon}}_{1} & =-\frac{1}{\pi} \sqrt{\frac{a}{\mu}} \int_{0}^{2 \pi}(1-e \cos E)^{2} F_{R}+\left(1-\sqrt{1-e^{2}}\right)(\overline{\dot{\omega}}+\overline{\dot{\Omega}}) \\
& +2 \sqrt{1-e^{2}} \sin ^{2}\left(\frac{i}{2}\right) \overline{\dot{\Omega}}
\end{aligned}
$$

Substituting the Fourier series for the thrust vector components into the averaged Gauss equations, we encounter the orthogonality conditions:

$$
\begin{gathered}
\int_{0}^{L} \cos n x \cos m x d x= \begin{cases}0 & n \neq m \\
\frac{L}{2} & n=m \neq 0 \\
L & n=m=0\end{cases} \\
\int_{0}^{L} \sin n x \sin m x d x= \begin{cases}0 & n \neq m, n=0, \text { or } m=0 \\
\frac{L}{2} & n=m \neq 0\end{cases}
\end{gathered}
$$

This orthogonality eliminates all but the zeroth-, first-, and/or second-order coefficients of each thrust-acceleration Fourier series. Thus, the average rates of change of the orbital elements $a, e, i, \Omega, \omega$, and $\epsilon_{1}$ are only dependent on the 14 Fourier coefficients $\alpha_{0}^{R, E}, \alpha_{1}^{R, E}$, $\alpha_{2}^{R, E}, \beta_{1}^{R, E}, \alpha_{0}^{S, E}, \alpha_{1}^{S, E}, \alpha_{2}^{S, E}, \beta_{1}^{S, E}, \beta_{2}^{S, E}, \alpha_{0}^{W, E}, \alpha_{1}^{W, E}, \alpha_{2}^{W, E}, \beta_{1}^{W, E}$, and $\beta_{2}^{W, E}$, regardless of the order of the original thrust Fourier series. Henceforth, we will drop the superscript $E$, as all thrust-acceleration Fourier series will be expanded in eccentric anomaly:

$$
\begin{aligned}
& \overline{\dot{a}}=2 \sqrt{\frac{a^{3}}{\mu}}\left[\frac{1}{2} e \beta_{1}^{R}+\sqrt{1-e^{2}} \alpha_{0}^{S}\right] \\
& \overline{\dot{e}}=\sqrt{\frac{a}{\mu}} \sqrt{1-e^{2}}\left[\frac{1}{2} \sqrt{1-e^{2}} \beta_{1}^{R}+\alpha_{1}^{S}-\frac{3}{2} e \alpha_{0}^{S}-\frac{1}{4} e \alpha_{2}^{S}\right] \\
& \bar{i}=\sqrt{\frac{a}{\mu}} \frac{1}{\sqrt{1-e^{2}}}\left[\frac{1}{2}\left(1+e^{2}\right) \cos \omega \alpha_{1}^{W}-\frac{3}{2} e \cos \omega \alpha_{0}^{W}\right. \\
& \left.-\frac{1}{2} \sqrt{1-e^{2}} \sin \omega \beta_{1}^{W}-\frac{1}{4} e \cos \omega \alpha_{2}^{W}+\frac{1}{4} e \sqrt{1-e^{2}} \sin \omega \beta_{2}^{W}\right] \\
& \overline{\dot{\Omega}}=\sqrt{\frac{a}{\mu}} \frac{\csc i}{\sqrt{1-e^{2}}}\left[\frac{1}{2} \sqrt{1-e^{2}} \cos \omega \beta_{1}^{W}+\frac{1}{2}\left(1+e^{2}\right) \sin \omega \alpha_{1}^{W}\right. \\
& \left.-\frac{3}{2} e \sin \omega \alpha_{0}^{W}-\frac{1}{4} e \sqrt{1-e^{2}} \cos \omega \beta_{2}^{W}-\frac{1}{4} e \sin \omega \alpha_{2}^{W}\right]
\end{aligned}
$$




$$
\begin{aligned}
\overline{\dot{\omega}} & =\sqrt{\frac{a}{\mu}} \frac{1}{e}\left[-\frac{1}{2} \sqrt{1-e^{2}} \alpha_{1}^{R}+e \sqrt{1-e^{2}} \alpha_{0}^{R}\right. \\
& \left.+\frac{1}{2}\left(2-e^{2}\right) \beta_{1}^{S}-\frac{1}{4} e \beta_{2}^{S}\right]-\cos i \overline{\dot{\Omega}} \\
\overline{\dot{\epsilon}}_{1} & =\sqrt{\frac{a}{\mu}}\left[\left(-2-e^{2}\right) \alpha_{0}^{R}+2 e \alpha_{1}^{R}-\frac{1}{2} e^{2} \alpha_{2}^{R}\right] \\
& +\left(1-\sqrt{1-e^{2}}\right)(\overline{\dot{\omega}}+\overline{\dot{\Omega}})+2 \sqrt{1-e^{2}} \sin ^{2}\left(\frac{i}{2}\right) \overline{\dot{\Omega}}
\end{aligned}
$$

The assumption of a thrust-acceleration vector specified over only one orbit period is not strictly necessary; the same averaging method can be used with acceleration functions specified over arbitrary lengths, simply by substituting Eqs. (12-17) and averaging the Gauss equations over the full interval. An example of this is presented in Sec. V. In general, when $L=m \pi$, the zeroth, $(m / 2)$ th, and $m$ th coefficients will remain, with fractional indices required in the original Fourier series when $m$ is not an even integer. However, the averaging assumption may become less valid for aperiodic control laws of long duration, for which the orbit changes significantly from start to finish.

Equations (36-41) contain singularities in the case of zero eccentricity or inclination, due to singularities in the Gauss equations. To avoid numerical difficulties when evaluating trajectories that closely approach circular or equatorial orbits, we substitute the variables

$$
\begin{gathered}
h_{1}=e \sin (\Omega+\omega) \\
k_{1}=e \cos (\Omega+\omega) \\
h_{2}=\sin i \sin \Omega \\
k_{2}=\sin i \cos \Omega
\end{gathered}
$$

The averaged differential equations for the new variables can be derived using the preceding approach. These substitutions are effective for near-zero eccentricity and inclination; however, we find inaccuracies when integrating trajectories that pass through exactly circular orbits, indicating that our averaging analysis must be reconsidered for this case. A deeper analysis of these singularities will be carried out in the future.

\section{Agreement with Newtonian Equations}

In the following, we present several examples to indicate the veracity of our analytical result. These simulations and comparisons are to provide additional insight into this result. Additional numerical verifications of these results were made but are not reported here. To verify the averaged secular equations, we first consider a simple control law: a step-acceleration function in the circumferential direction only, with two burns and coast arcs, as shown in Fig. 1 .

The Fourier series for this step function is determined by Eqs. (22-24):

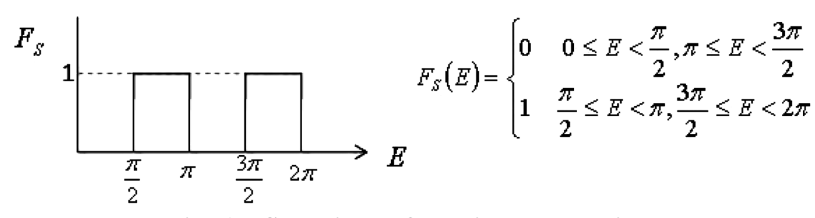

Fig. 1 Step circumferential acceleration.

$$
\begin{gathered}
F_{S}=\sum_{k=0}^{\infty} \alpha_{k}^{S} \cos (k E)+\beta_{k}^{S} \sin (k E) \\
\alpha_{0}^{S}=\frac{1}{2 \pi} \int_{0}^{2 \pi} F_{S} d E=\frac{1}{2} \\
\alpha_{k}^{S}=\frac{1}{\pi} \int_{0}^{2 \pi} F_{S} \cos (k E) d E=0
\end{gathered}
$$

$$
\beta_{k}^{S}=\frac{1}{\pi} \int_{0}^{2 \pi} F_{S} \sin (k E) d E= \begin{cases}-\frac{4}{k \pi} & k=2,6,10, \ldots \\ 0 & \text { otherwise }\end{cases}
$$

Figure 2 compares this Fourier series, numerically evaluated up to order $100 \overline{0}$, to the series of only the five terms that appear in the averaged secular equations. Clearly, there is considerable variation between these two representations of the periodic step-acceleration control law. (All dimensions herein are normalized to a standard gravitational parameter $\mu=1$; thus, figure units are not stated.)

Figure $\underline{3}$ describes the osculating orbital elements of an example spacecraft subjected to these thrusts. Equations (36-41) with $\alpha_{0}^{S}, \alpha_{1}^{S}$, $\alpha_{2}^{S}, \beta_{1}^{S}$, and $\beta_{2}^{S}$ were integrated using a Runge-Kutta method to estimate the trajectory over 10 orbits. For comparison, the Newtonian equations (1) and (2) were also integrated using the Fourier series up to order 1000 . The two methods determined very similar orbital trajectories.

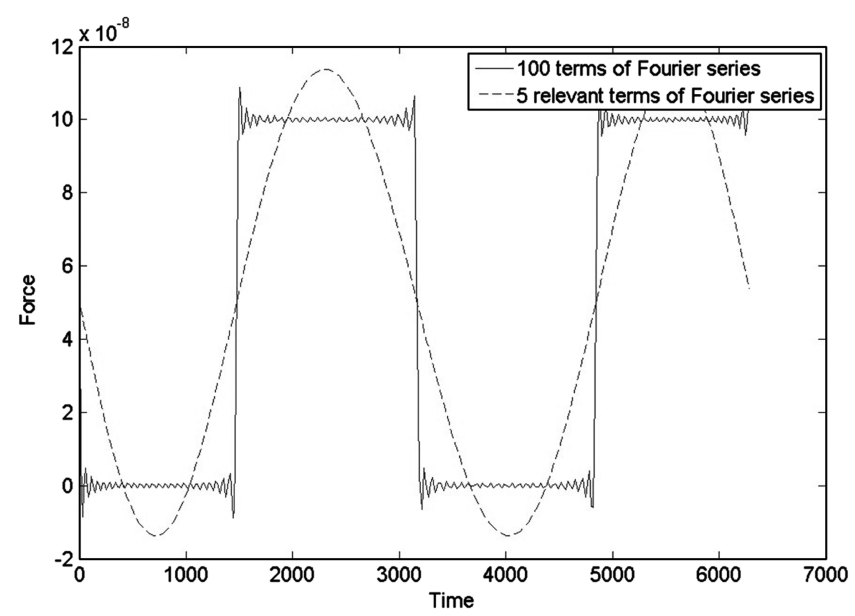

Fig. 2 Fourier series for step circumferential acceleration.
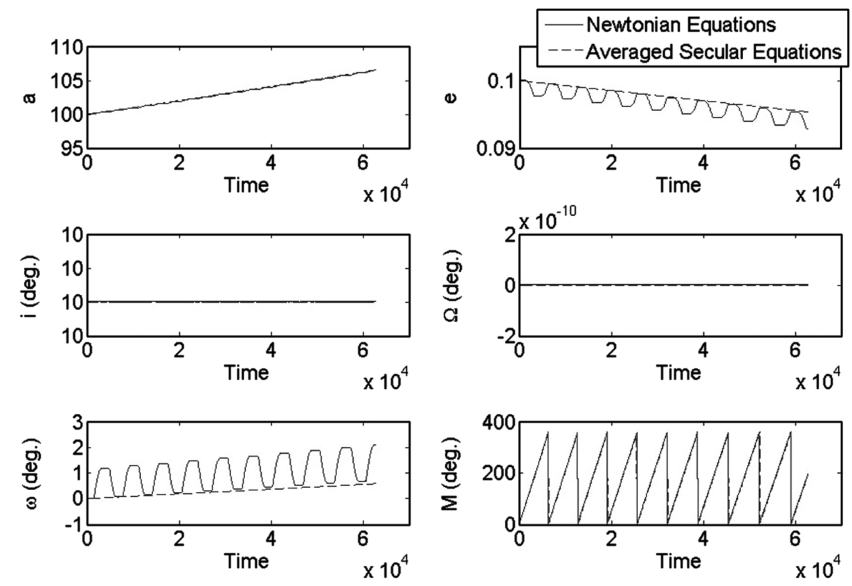

Fig. 3 Osculating orbital elements of spacecraft subject to step circumferential acceleration. 
Another case of interest is acceleration with constant magnitude but varying direction, as on a spacecraft with one gimbaled thruster. As a simple example of this case, we consider an acceleration that oscillates sinusoidally between the radial and circumferential directions, as shown in Fig. 4. The Fourier series for the components of this acceleration vector are immediate: $\alpha_{1}^{R}=1, \alpha_{0}^{S}=1$, and $\alpha_{1}^{S}=-1$, and all other coefficients are zero.

Figure 5 shows the trajectory resulting from this acceleration, as determined by both the Newtonian equations and the averaged secular equations. Again, there is close agreement between the two methods.

Next, we consider a more complex control law. Figures $\underline{6}-\underline{8}$ describe the trajectory of an example system for which the thrustacceleration Fourier coefficients were randomly selected up to order 10 within the range of $-2.5 \mathrm{e}-7$ to $2.5 \mathrm{e}-7$. Figure $\underline{6}$ compares the time histories of the osculating orbital elements as determined by both methods. We note that there is precise agreement between the Newtonian equations and the averaged secular equations for the first several orbits. In the later orbits, there is some drift, most noticeable in mean anomaly and argument of periapsis, due to higher-order effects not captured in the averaging process and to mismatch between the average initial conditions and the secular initial conditions. Correction of this drift will be addressed in Sec. VI.

Figure 7 compares one component of this acceleration vector over three orbits with the first five terms of its Fourier series expansion (i.e., the terms that appear in the secular equations).

Figure 8 shows the eccentric anomaly over the 15 -orbit time span. Note that the eccentric anomaly shifts slightly as the nominal orbit evolves. This implies that the original control law has a changing time variation as the orbit evolves, even though the coefficients may stay constant.

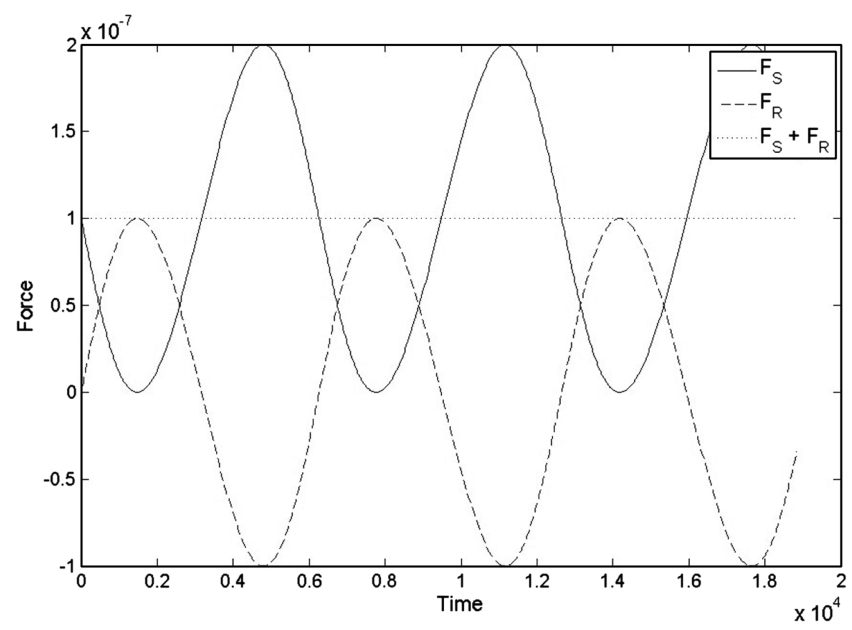

Fig. 4 Constant-magnitude acceleration.
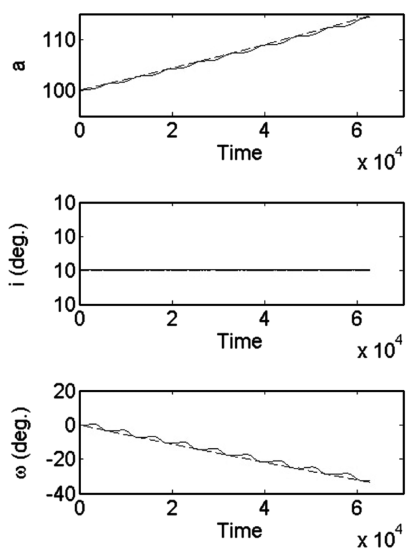

Fig. 5 Osculating orbital elements of spacecraft subject to constantmagnitude acceleration.
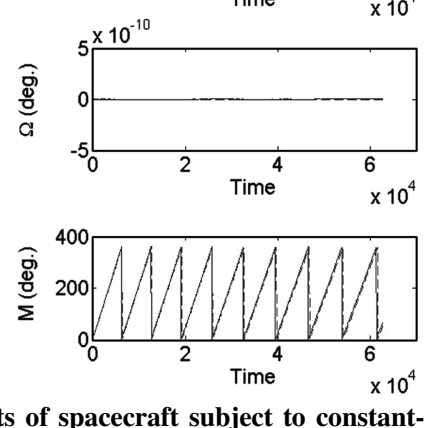
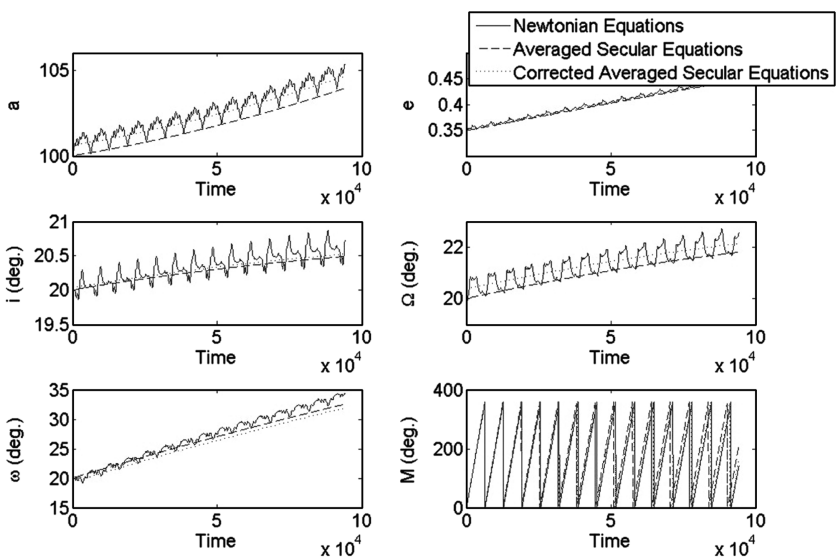

Fig. 6 Randomly generated acceleration.

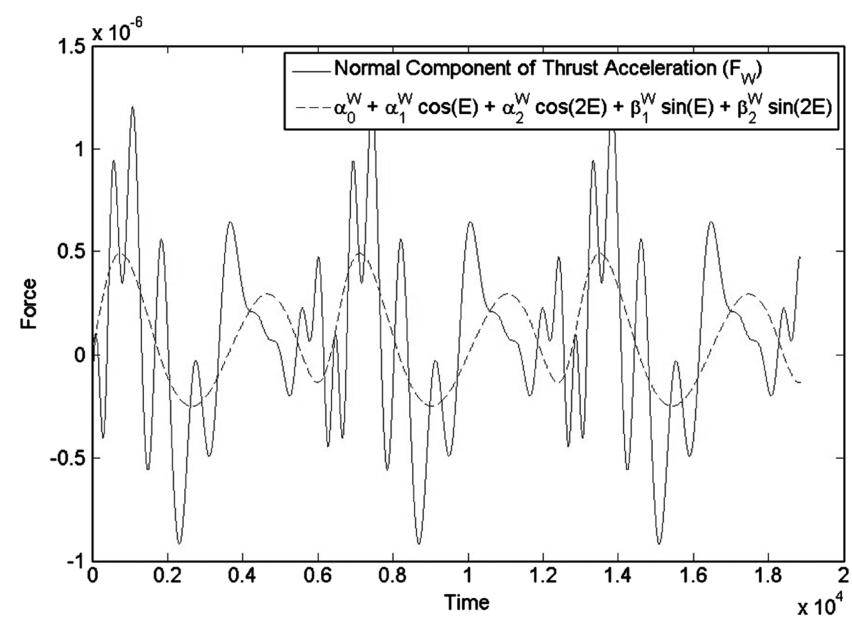

Fig. 7 Normal component of the acceleration vector.

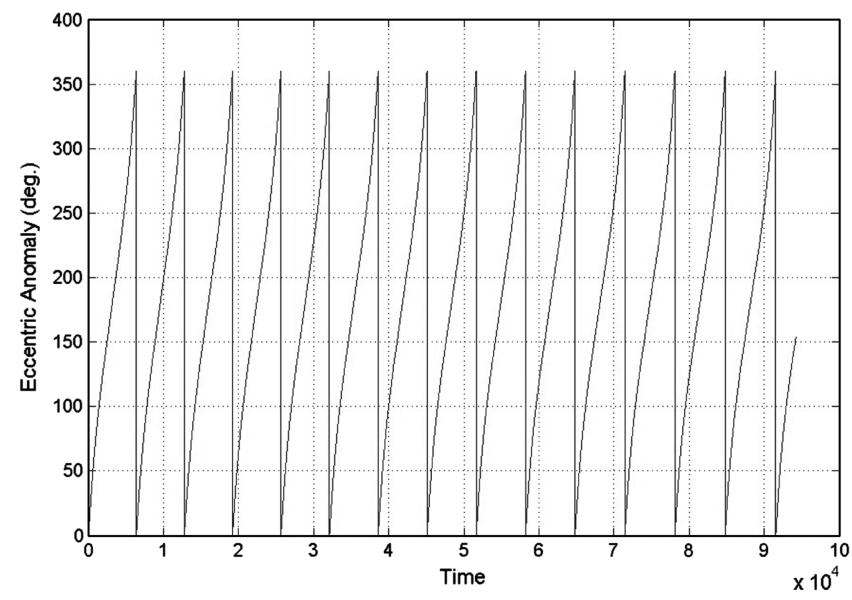

Fig. 8 Eccentric anomaly vs time.

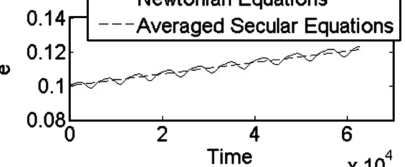

Figures 9 and 10 show an example of the same method applied to a control law with a period longer than $2 \pi$. In this example, the acceleration function is defined on the interval $(0,6 \pi)$, with randomly selected Fourier coefficients in the range of $-2.5 \mathrm{e}-7$ to $2.5 \mathrm{e}-7$ and dimensions normalized to $\mu=1$. The 14 coefficients that remain in the averaged secular equations in this case are $\alpha_{0}^{R}, \alpha_{3}^{R}$, $\alpha_{6}^{R}, \beta_{3}^{R}, \alpha_{0}^{S}, \alpha_{3}^{S}, \alpha_{6}^{S}, \beta_{3}^{S}, \beta_{6}^{S}, \alpha_{0}^{W}, \alpha_{3}^{W}, \alpha_{6}^{W}, \beta_{3}^{W}$, and $\beta_{6}^{W}$. Figure 9 shows the osculating orbital elements over 20 orbits. Figure 10 shows the normal component of the acceleration vector and the first five terms of its Fourier series over the first nine orbits or the first three cycles of the periodic acceleration. 

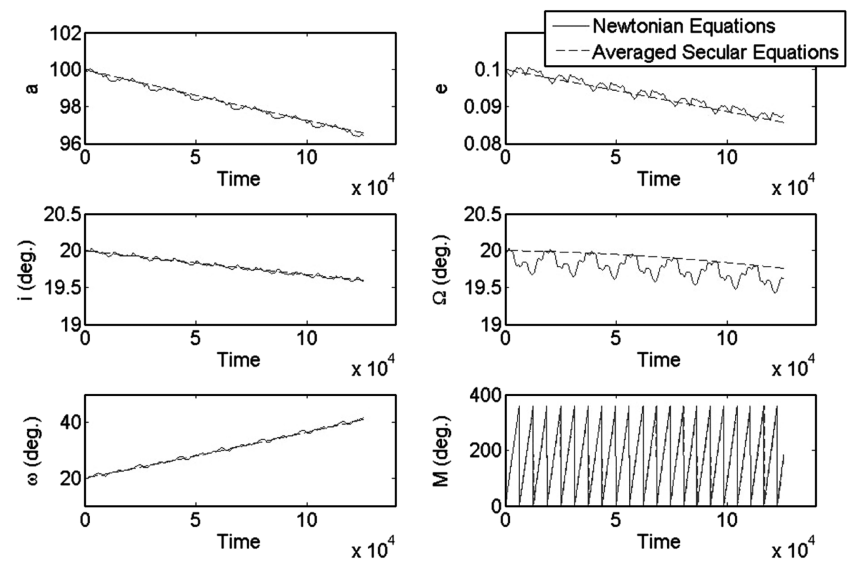

Fig. 9 Randomly generated acceleration with period $6 \pi$.

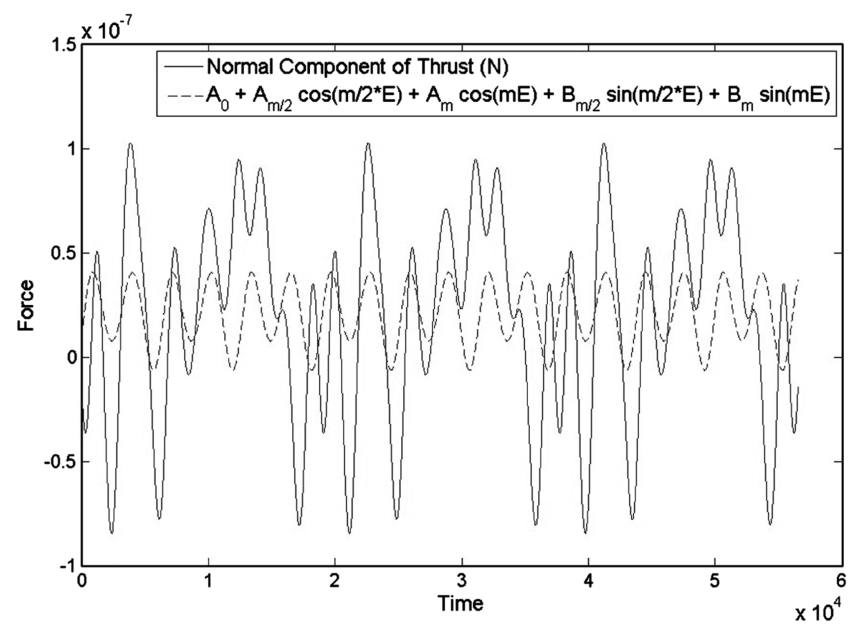

Fig. 10 Normal component of the $6 \pi$ periodic acceleration vector over nine orbits.

\section{Offset Correction}

Average trajectories calculated using Eqs. (36-41) show the correct trends in the evolution of the osculating orbital elements and are good approximations of the true trajectories. However, they may be offset from the true averages and may diverge from true trajectories over many orbits. This may be partially due to higherorder effects not captured in the averaging method, but it may also be due to nontrivial periodic components, which can shift the mean value of the state from the initial condition. This initial condition offset can be corrected by the addition of an averaged periodic term to the initial conditions of the secular equation for each orbital element.

At any time $t$, the true value of any orbital element can be expressed as the sum of a secular term and a periodic term. The periodic term repeats itself over each orbit:

$$
œ(t)=œ_{0}+\dot{\bar{\propto}} t+œ_{p}(t)
$$

The true average value of the orbital element over one orbit is thus

$$
\bar{œ}=œ_{0}+\frac{T}{2} \dot{\overline{\mathfrak{e}}}+\overline{\mathfrak{\aleph}}_{p}
$$

where $T$ represents the period, $\mathbf{x}$ represents a vector of the six orbital elements, and $\dot{\overline{\mathrm{e}}}=\bar{f}(\mathbf{x})$ represents Eqs. $(\underline{36}-41)$.

The time derivative of Eq. (50) provides a differential equation for the periodic term:

$$
\dot{\mathfrak{e}}_{p}=f(\mathbf{x}, t)-\bar{f}(\mathbf{x})
$$

We substitute the nominal initial condition $\mathbf{x}_{0}$ for the true orbital element vector, knowing that the corrections are of higher order, and perform the quadrature for $œ_{p}$ :

$$
œ_{p}=\int_{0}^{t} f\left(\mathbf{x}_{0}, \tau\right) \mathrm{d} \tau-\bar{f}\left(\mathbf{x}_{0}\right) t
$$

This periodic term can be averaged over one orbit:

$$
\bar{\aleph}_{p}=\frac{1}{2 \pi} \int_{0}^{2 \pi}\left[\int_{0}^{t} f\left(\mathbf{x}_{0}, \tau\right) d \tau-\bar{f}\left(\mathbf{x}_{0}\right) t\right] d M
$$

For compatibility with the form of the preceding Gauss equations, the two integrals are shifted to eccentric anomaly:

$$
\begin{aligned}
\bar{\propto}_{p} & =\frac{1}{2 \pi} \int_{0}^{2 \pi}\left[\int_{E_{0}}^{E} \frac{1}{n}\left(1-e \cos E^{\prime}\right) f\left(\mathbf{x}_{0}, E^{\prime}\right) d E^{\prime}\right](1-e \cos E) d E \\
& -\frac{\pi}{n} \bar{f}\left(\mathbf{x}_{0}\right)
\end{aligned}
$$

where $n=\sqrt{\mu / a^{3}}$ is the mean motion. Note that $\pi / n=T / 2$. This expression for $\bar{\propto}_{p}$ can be substituted into Eq. (51) to determine the average value of the orbital element over the first period:

$$
\begin{aligned}
\bar{\propto} & =œ_{0}+\frac{1}{2 \pi} \int_{0}^{2 \pi}\left[\int_{E_{0}}^{E} \sqrt{\frac{a^{3}}{\mu}}\left(1-e \cos E^{\prime}\right) f\left(\mathbf{x}_{0}, E^{\prime}\right) d E^{\prime}\right] \\
& \times(1-e \cos E) d E
\end{aligned}
$$

To correct the initial conditions for the averaged secular equations, Eq. (55) is substituted into Eq. (50) at $t=0$. The averaged secular equations, thus initialized, yield a more accurate average of the true periodic trajectory:

$$
\begin{aligned}
& \propto(0)=œ_{0}+\frac{1}{2 \pi} \int_{0}^{2 \pi}\left[\int_{E_{0}}^{E} \frac{1}{n}\left(1-e \cos E^{\prime}\right) f\left(\mathbf{x}, E^{\prime}\right) d E^{\prime}\right] \\
& \times(1-e \cos E) d E-\frac{T}{2} \bar{f}(\mathbf{x})
\end{aligned}
$$

To calculate a value for the averaged periodic correction term, we substitute the Fourier series in eccentric anomaly for the acceleration component terms in the Gauss equations. To avoid infinite series in the solution, we include only the 14 terms of the Fourier series for which the coefficients appear in the averaged secular equations, as they have been shown to have the most significant effect on the trajectory dynamics. Assuming zero-eccentric-anomaly initial conditions, the correction terms are

$$
\begin{gathered}
\bar{a}_{p}=\frac{a^{3}}{\mu}\left[e\left((2+e) \alpha_{0}^{R}+\frac{1}{2} \alpha_{1}^{R}-\left(\frac{1}{2} e+\frac{2}{3}\right) \alpha_{2}^{R}+\pi \beta_{1}^{R}\right)\right. \\
\left.+\sqrt{1-e^{2}}\left(2 \pi \alpha_{0}^{S}+(2+e) \beta_{1}^{S}+\beta_{2}^{S}\right)\right]-\frac{T}{2} \overline{\dot{a}}\left(\mathbf{x}_{0}\right)
\end{gathered}
$$

$$
\begin{aligned}
\bar{e}_{p} & =\frac{1}{2} \frac{a^{2}}{\mu}\left[\left(1-e^{2}\right)\left((2+e) \alpha_{0}^{R}+\frac{1}{2} \alpha_{1}^{R}-\left(\frac{1}{2} e+\frac{2}{3}\right) \alpha_{2}^{R}+\pi \beta_{1}^{R}\right)\right. \\
& +\sqrt{1-e^{2}}\left(-3 e \pi \alpha_{0}^{S}+2 \pi \alpha_{1}^{S}-\frac{1}{2} e \pi \alpha_{2}^{S}+\left(1-\frac{5}{4} e^{2}-\frac{8}{3} e\right) \beta_{1}^{S}\right. \\
& \left.\left.+\left(-\frac{5}{8} e+\frac{8}{3}\right) \beta_{2}^{S}\right)\right]-\frac{T}{2} \overline{\dot{e}}\left(\mathbf{x}_{0}\right)
\end{aligned}
$$




$$
\begin{aligned}
\bar{i}_{p} & =\frac{1}{2} \frac{a^{2}}{\mu} \frac{1}{\sqrt{1-e^{2}}}\left[\alpha_{0}^{W}\left(\sqrt{1-e^{2}} \sin \omega\left(-1-\frac{1}{2} e\right)-3 \pi e \cos \omega\right)\right. \\
& +\alpha_{1}^{W}\left(\pi \cos \omega\left(1+e^{2}\right)+\sqrt{1-e^{2}} \sin \omega\left(\frac{2}{3} e+\frac{1}{4} e^{2}-\frac{1}{2}\right)\right) \\
& +\alpha_{2}^{W}\left(\sqrt{1-e^{2}} \sin \omega\left(\frac{2}{3}+\frac{5}{8} e\right)-\frac{1}{2} \pi e \cos \omega\right) \\
& +\beta_{1}^{W}\left(\cos \omega\left(\frac{1}{2}-\frac{8}{3} e-\frac{3}{4} e^{2}\right)-\pi \sqrt{1-e^{2}} \sin \omega\right) \\
& +\beta_{2}^{W}\left(\cos \omega\left(\frac{4}{3}-\frac{9}{8} e+\frac{4}{3} e^{2}+\frac{1}{2} e^{3}\right)\right. \\
& \left.\left.+\frac{1}{2} \pi e \sqrt{1-e^{2}} \sin \omega\right)\right]-\frac{T}{2} \bar{i}\left(\mathbf{x}_{0}\right)
\end{aligned}
$$

$$
\begin{aligned}
\bar{\Omega}_{p} & =\frac{1}{2} \frac{a^{2}}{\mu} \frac{\csc i}{\sqrt{1-e^{2}}}\left[\operatorname { c o s } \omega \sqrt { 1 - e ^ { 2 } } \left(\alpha_{0}^{W}\left(2+\frac{1}{2} e\right)\right.\right. \\
& +\alpha_{1}^{W}\left(-\frac{1}{4} e^{2}+\frac{1}{2}-\frac{2}{3} e\right)+\alpha_{2}^{W}\left(-\frac{5}{8} e-\frac{2}{3}\right) \\
& \left.+\pi \beta_{1}^{W}-\frac{1}{2} e \pi \beta_{2}^{W}\right)+\sin \omega\left(\pi \alpha_{1}^{W}\left(1+e^{2}\right)-\frac{7}{2} \pi e \alpha_{2}^{W}\right. \\
& \left.\left.+\beta_{1}^{W}\left(\frac{1}{2}-\frac{8}{3} e-\frac{3}{4} e^{2}\right)+\beta_{2}^{W}\left(\frac{4}{3}-\frac{9}{8} e+\frac{4}{3} e^{2}+\frac{1}{2} e^{3}\right)\right)\right] \\
& -\frac{T}{2} \dot{\bar{\Omega}}\left(\mathbf{x}_{0}\right)
\end{aligned}
$$

$$
\begin{aligned}
\bar{\omega}_{p} & =\frac{1}{2} \frac{a^{2}}{\mu} \frac{1}{e}\left[\alpha_{0}^{S}\left(4-\frac{3}{2} e-2 e^{2}-e^{3}\right)+\alpha_{1}^{S}\left(\pi-\frac{2}{3} e-\frac{3}{4} e^{2}\right)\right. \\
& +\alpha_{2}^{S}\left(-\frac{4}{3}-\frac{9}{8} e+\frac{2}{3} e^{2}+\frac{1}{2} e^{3}\right) \pi \beta_{1}^{S}\left(2-e^{2}\right)-\frac{1}{2} \pi \beta_{2}^{S} \\
& +2 e \pi \sqrt{1-e^{2}} \alpha_{0}^{R}-\pi \sqrt{1-e^{2}} \alpha_{1}^{R} \\
& \left.+\beta_{1}^{R} \sqrt{1-e^{2}}\left(-\frac{1}{2}+2 e+e^{2}\right)\right]-\cos i \bar{\Omega}_{p} \\
& +\frac{T}{2} \overline{\dot{\Omega}}\left(\mathbf{x}_{0}\right)-\frac{T}{2} \overline{\dot{\omega}}\left(\mathbf{x}_{0}\right)
\end{aligned}
$$

$$
\begin{aligned}
\bar{\epsilon}_{1 p} & =-\frac{a^{2}}{\mu}\left[\pi \alpha_{0}^{R}\left(2+e^{2}\right)-2 \pi e \alpha_{1}^{R}+\frac{1}{2} \pi e^{2} \alpha_{2}^{R}\right. \\
& \left.+\beta_{1}^{R}\left(2-e+\frac{2}{3} e^{2}\right)\right]+\left(1-\sqrt{1-e^{2}}\right) \\
& \times\left(\omega_{p}+\Omega_{p}+\frac{T}{2} \overline{\dot{\omega}}\left(\mathbf{x}_{0}\right)+\frac{T}{2} \overline{\dot{\Omega}}\left(\mathbf{x}_{0}\right)\right) \\
& +2 \sqrt{1-e^{2}} \sin ^{2} \frac{i}{2}\left(\Omega_{p}+\frac{T}{2} \overline{\dot{\Omega}}\left(\mathbf{x}_{0}\right)\right)-\frac{T}{2} \overline{\dot{\epsilon}}_{1}\left(\mathbf{x}_{0}\right)
\end{aligned}
$$

Figure 6 shows the effect of these corrections on the system with randomly selected Fourier coefficients. The corrected initial conditions shift the estimated average trajectory to more accurately reflect the true average trajectory. They also can reduce the divergence between the true and average trajectories, most noticeably in the plot of mean anomaly. Some drift remains, however, due to higher-order effects. This can be seen in the argument of periapsis: the corrected trajectory closely approximates the true average trajectory over the first few orbits, but diverges as higher-order effects accumulate over several orbits.

\section{Conclusions}

A novel method to efficiently evaluate the trajectory dynamics resulting from low-thrust propulsion was developed. The thrustacceleration vector components were represented as Fourier series in eccentric anomaly, then Gauss's variational equations were averaged over one orbit and simplified by the orthogonality conditions. The resulting secular equations were a function of 14 of the thrust Fourier coefficients, regardless of the order of the original Fourier series. Thus, a general thrust profile was reduced to a set of only 14 parameters.

The averaged variational equations in the 14 coefficients were shown to accurately determine spiral trajectories resulting from continuous or discontinuous low-thrust propulsion over many orbits, as compared with numerical integration of the full Newtonian equations of motion. Offsets of the averaged trajectory due to initial conditions were corrected by addition of an averaged periodic term.

Unlike many special-case solutions, this analytical method is not limited to constant-magnitude or constant-direction thrust. Applications of this method include low-thrust spacecraft targeting and optimal control problems, which could be solved efficiently using the averaged secular equations.

\section{Acknowledgments}

This material is based upon work supported under a National Science Foundation Graduate Research Fellowship. Any opinions, findings, conclusions, or recommendations expressed in this publication are those of the authors and do not necessarily reflect the views of the National Science Foundation.

\section{References}

[1] Forbes, G. F., "The Trajectory of a Powered Rocket in Space," Journal of the British Interplanetary Society, Vol. 9, No. 2, 1950, pp. 75-79.

[2] Tsu, T. C., "Interplanetary Travel by Solar Sail," Journal of the American Rocket Society, Vol. 29, No. 6, 1959, pp. 422-427.

[3] Pinkham, G., "Reference Solution for Low Thrust Trajectories," Journal of the American Rocket Society, Vol. 32, No. 5, 1962, pp. 775776.

[4] Petropoulos, A. E., Longuski, J. M., and Vinh, N. X., "Shape-Based Analytic Representations of Low-Thrust Trajectories for GravityAssist Applications," American Astronautical Society, Paper 99-337, Aug. 1999.

[5] Petropoulos, A. E., and Longuski, J. M., "Shape-Based Algorithm for Automated Design of Low-Thrust, Gravity-Assist Trajectories," Journal of Spacecraft and Rockets, Vol. 41, No. 5, 2004, pp. 787-796. doi:10.2514/1.13095

[6] Tsien, H. S., "Take-Off from Satellite Orbit," Journal of the American Rocket Society, Vol. 23, No. 4, 1953, pp. 233-236.

[7] Battin, R. H., An Introduction to the Mathematics and Methods of Astrodynamics, AIAA Education Series, AIAA, New York, 1987, pp. 408-415.

[8] Prussing, J., and Coverstone-Carroll, V., "Constant Radial Thrust Acceleration Redux," Journal of Guidance, Control, and Dynamics, Vol. 21, No. 3, 1998, pp. 516-518. doi: $10.2514 / 2.7609$

[9] Markopoulos, N., "Explicit, Near-Optimal Guidance For PowerLimited Escape from a Circular Orbit," AIAA Guidance, Navigation and Control Conference, AIAA Paper 96-3706, July 1996.

[10] Lawden, D. F., "Optimal Intermediate-Thrust Arcs in a Gravitational Field," Astronautica Acta, Vol. 8, 1962, pp. 106-123.

[11] Bishop, R. H., and Azimov, D. M., "Analytical Space Trajectories for Extremal Motion with Low-Thrust Exhaust-Modulated Propulsion," Journal of Spacecraft and Rockets, Vol. 38, No. 6, 2001, pp. 897-903. doi:10.2514/2.3761

[12] Marec, J. P., and Vinh, N. X., "Optimal Low-Thrust, Limited Power Transfers Between Arbitrary Elliptical Orbits," Acta Astronautica, Vol. 4, Nos. 5-6, 1977, pp. 511-540. doi:10.1016/0094-5765(77)90106-0

[13] Kluever, C. A., and Oleson, S. R., "Direct Approach for Computing Near-Optimal Low-Thrust Earth-Orbit Transfers," Journal of Spacecraft and Rockets, Vol. 35, No. 4, 1998, pp. 509-515. doi: $10.2514 / 2.3360$

[14] Kluever, C. A., "Simple Guidance Scheme for Low-Thrust Orbit Transfers," Journal of Guidance, Control, and Dynamics, Vol. 21, 
No. 6, 1998, pp. 1015-1017. doi: $10.2514 / 2.4344$

[15] Kluever, C. A., "Low-Thrust Orbit Transfer Guidance Using an Inverse Dynamics Approach," Journal of Guidance, Control, and Dynamics, Vol. 18, No. 1, 1995, pp. 187-189. doi: $10.2514 / 3.56676$

[16] Kluever, C. A., and Shaughnessy, D. J., "Trajectory-Tracking Guidance Law for Low-Thrust Earth-Orbit Transfers," Journal of Guidance, Control, and Dynamics, Vol. 23, No. 4, 2000, pp. 754-756. doi:10.2514/2.4597

[17] Ilgen, M. R., "Low Thrust OTV Guidance Using Lyapunov Optimal Feedback Control Techniques," AAS/AIAA Astrodynamics Specialist Conference, American Astronautical Society Paper 93-680, Aug. 1993.

[18] Gurfill, P., "Nonlinear Feedback Control of Low-Thrust Orbital Transfer in a Central Graviational Field," Acta Astronautica, Vol. 60, Nos. 8-9, 2007, pp. 631-648. doi:10.1016/j.actaastro.2006.10.001

[19] Petropoulos, A. E., "Some Analytic Integrals of the Averaged Variational Equations for a Thrusting Spacecraft," Interplanetary
Network Progress Rept. 42-150, Jet Propulsion Lab., California Inst. of Technology, Pasadena, CA, Aug. 2002, pp. 1-29.

[20] Edelbaum, T. N., "Optimum Power-Limited Orbit Transfer in Strong Gravity Fields," AIAA Journal, Vol. 3, No. 5, May 1965, pp. 921-925. doi: $10.2514 / 3.3016$

[21] Gao, Y., "Near-Optimal Very Low-Thrust Earth-Orbit Transfers and Guidance Schemes," Journal of Guidance, Control, and Dynamics, Vol. 30, No. 2, 2007, pp. 529-539. doi: $10.2514 / 1.24836$

[22] Gao, Y., "low-thrust Nonlinear Guidance by Tracking Mean Orbital Elements," Journal of Guidance, Control, and Dynamics, Vol. 31, No. 4, 2008, pp. 1103-1110. doi: $10.2514 / 1.31256$

[23] Danby, J. M. A., Fundamentals of Celestial Mechanics, 2nd ed. 5th printing, Willmann-Bell, Richmond, VA, 2003, pp. 327-328.

[24] Haberman, R., Applied Partial Differential Equations with Fourier Series and Boundary Value Problems, 4th ed., Pearson Prentice-Hall, Upper Saddle River, NJ, 2004, pp. 89-92. 